\title{
Chronic pelvic pain: comorbidity between chronic musculoskeletal pain and vulvodynia
}

\author{
G. Biasi ${ }^{1}$, V. Di Sabatino', A. Ghizzani ${ }^{2}$, M. Galeazzi ${ }^{1}$ \\ ${ }^{1}$ Rheumatology Unit, Policlinico Le Scotte, Medicine, Surgery and Neurosciences Department, \\ University of Siena; ${ }^{2}$ Obstetrics and Gynecology Unit, Policlinico Le Scotte, Medicine, \\ Surgery and Neurosciences Department, University of Siena, Italy
}

\begin{abstract}
SUMMARY
Chronic pelvic pain (CPP) is a common condition that has a major impact on the quality of life of both men and women. Male CPP is usually attributable to well-defined urogenital conditions (most frequently infectious/non infectious prostatic diseases) or musculoskeletal or bowel diseases, whereas the features of female CPP are much more complex and are of particular clinical and epidemiological importance. It is a multifactorial syndrome that can be due to diseases of the urogenital, gastrointestinal, or musculoskeletal systems, or to neurological or neuropsychiatric disorders. It is not always easy to identify its predominant pathogenesis, although it often occurs as a central sensitization syndrome triggered by an initial stimulus which is no longer detectable and only manifests itself clinically through pain. In this respect, there are some very interesting relationships between vulvodynia and fibromyalgic syndrome, as identified in a preliminary study of women with chronic musculoskeletal pain in which it was demonstrated that vulvar pain plays an important role, although it is often overlooked and undiagnosed.
\end{abstract}

Key words: Vulvodynia, Fibromyalgia, Pain.

Reumatismo, 2014; 66 (1): 87-91

\section{INTRODUCTION}

Ciser hronic pelvic pain (CPP), which is defined as a condition localized in the pelvic region lasting longer than three months, has a major impact on the quality of life of both men and women. It can cause functional disability, and requires appropriate pharmacological or surgical treatment. Male CPP is usually attributable to well-defined urogenital conditions (most frequently infectious/non infectious prostatic diseases) or musculoskeletal or bowel diseases (1), whereas the features of female CPP (defined as chronic pain affecting the pelvis and lower abdominal region that is not related to the menstrual cycle, sexual intercourse or pregnancy) (2) are much more complex and are of particular clinical and epidemiological importance.

\section{EPIDEMIOLOGY}

The epidemiological data vary widely and are difficult to interpret, although so far there has been a unanimous agreement on the diagnostic criteria. One of the most reliable studies was carried out in Great Britain and showed an annual prevalence of $3.8 \%$, which is very similar to that of bronchial asthma (3.7\%) and back pain $(4.1 \%)$ more frequently observed in women of childbearing age. Another study conducted in New Zealand showed a frequency of $25 \%$ in a sample of women aged 18-50 years, and an American survey indicated a frequency of $14.7 \%$ in the same age range (3).

\section{ETIOPATHOGENESIS}

CPP is a multifactorial syndrome that can be due to diseases related to urogenital, gastrointestinal, musculoskeletal systems, or to neuropsychiatric or neurological disorders (Tab. I).

When the initial pathology can be easily diagnosed on the basis of anamnestic and clinical data, it is possible to establish an effective and specific pharmacological or
Corresponding author: Giovanni Biasi Medicine, Surgery and Neurosciences Department

University of Siena, Italy

E-mail: gbocto@tin.it 
Table I - Causes of chronic pelvic pain.

\begin{tabular}{|l|}
\hline Gynecological causes \\
\hline Endometriosis \\
\hline Adenomyosis \\
\hline Chronic pelvic inflammatory disease \\
\hline Adherence syndromes \\
\hline Vaginal pain syndrome \\
\hline Vulvodynia \\
\hline Urological causes \\
\hline Interstitial cystitis \\
\hline Painful bladder syndrome \\
\hline Urethral syndrome \\
\hline Gastroenterological causes \\
\hline Irritable bowel syndrome \\
\hline Chronic inflammatory bowel diseases \\
\hline Celiac disease \\
\hline Diverticular disease - anorectal syndromes \\
\hline Musculoskeletal causes \\
\hline Fibromyalgia \\
\hline Abdominal wall myofascial syndromes \\
\hline $\begin{array}{l}\text { Degenerative/inflammatory sacroiliac and } \\
\text { coxofemoral joints }\end{array}$ \\
\hline $\begin{array}{l}\text { Degenerative/inflammatory lumbosacral spine } \\
\text { diseases (disk diseases, coccydynia) }\end{array}$ \\
\hline Pelvic floor myalgia (piriformis or anus levator \\
syndrome, postural abnormalities) \\
\hline Neurological causes \\
\hline Entrapment/compression syndromes \\
\hline Neuropathy (pudendal, iliohypogastric, ilioinguinal, \\
genitofemoral nerves) \\
\hline Psychopathological causes \\
\hline Personality disorders \\
\hline Anxious-depressive syndromes and sleep disorders \\
\hline Psycho-sexual disorders (abuse) \\
\hline
\end{tabular}

surgical rehabilitation, depending on the situation. However, in the presence of multiple factors, the predominant pathogenesis is not always easy to identify, although it often occurs as a central sensitization syndrome triggered by an initial stimulus which is no longer detectable, and only manifests itself clinically through pain. CPP is sometimes a single event, such as vaginal pain syndrome, pain bladder syndrome or vulvodynia, but it is more frequently an integral part of a more complex clinical picture as in the case of the fibromyalgic syndrome (FMS).

It is beyond the scope of this review to discuss all of the possible causes but, when making a differential diagnosis of pain in the pelvic region, rheumatologists should always consider all of the conditions listed in table I.

As in all cases of chronic pain, it is very important to take a thorough social and family history, including behavioral aspects, and to try to obtain a detailed description of the history of the pain and the factors modulating its intensity and quality, any associated symptoms, and the effects of any previous treatment. As comorbidities are not always obvious or not reported spontaneously by patients, they should be actively sought, especially in the presence of central sensitization, because any failure or delay in detecting a nociceptive event may contribute to the genesis and/or self-maintenance of the syndrome itself, and decrease the efficacy of any treatment.

In this respect there are many interesting relationships between vulvodynia and FMS as identified in a preliminary study of women with chronic musculoskeletal pain in which it was shown that vulvar pain plays an important role that is often overlooked and undiagnosed (4).

\section{Vulvodynia}

In 2003, the International Society for the Study of Vulvovaginal Disease (ISSVD) proposed a new classification of vulvar pain syndromes, and defined vulvodynia as vulvar discomfort, most often described as burning pain that occurs in the absence of detectable or specific signs (5).

The prevalence of vulvodynia is about $10-16 \%$ of the female population, but the problem is very often misunderstood and underestimated (6). The first symptoms usually occur at age 18-25 (7), but the average age at diagnosis is 32 years and the women are generally nulliparous (8). The vulvar pain is considered chronic if it lasts for at least three months.

The 2003 ISSVD classification divided vulvar pain into two diagnostic groups: the first involves specific problems such as infection, inflammation, cancer and neurological diseases; the second covers vulvar pain that cannot be traced to any specific cause and is called vulvodynia. Vulvodynia may be localized or generalized (Fig. 1). 
Localized vulvodynia is the most common form, with pain reported only at the level of the vulvar vestibule (usually in the posterior region of the vagina). It is considered primary when the pain occurred during the first sexual intercourse, and secondary when it appears after a period of normal sexual activity without pain. In the case of generalized vulvodynia, the pain is present throughout the vulvar region. In both forms, the vulvar pain can be provoked by slight pressure or be unprovoked $(5,6)$. Localized vulvodynia is often associated with trauma or inflammatory processes, whereas the generalized form seems to be more the result of a neuropathic problem and/or central sensitization.

As in the case of other diseases characterized by chronic pain, its etiology is unknown, although the various hypotheses include:

1) anomalies in embryonic development, such as a defect in the primitive urogenital sinus;

2) genetic and immunological disorders;

3) a neuropathic pain syndrome with elements related to a process of sensitization of the central nervous system (the most widely accepted hypothesis) (9).

The diagnosis is essentially clinical and based on Friederich's diagnostic criteria (10):

1. Localized intense pain upon a light touch applied to the vestibule or during attempts to penetrate;

2. Tenderness to pressure localized in the vestibule;

3. Absence of objective findings except for varying degrees of erythema.

The diagnosis is by exclusion, as this pain syndrome has no identifiable cause. It is therefore crucial to take a thorough medical history concerning the duration of the pain, its characteristics, previous treatments, allergies, surgery, sexual activity, a history of abuse, and any concomitant chronic pain diseases (fibromyalgia, chronic fatigue syndrome, interstitial cystitis, low back pain, migraine) $(11,12)$.

The subsequent physical examination involves a visual inspection (the visible characteristics of vulvodynia are erythema and

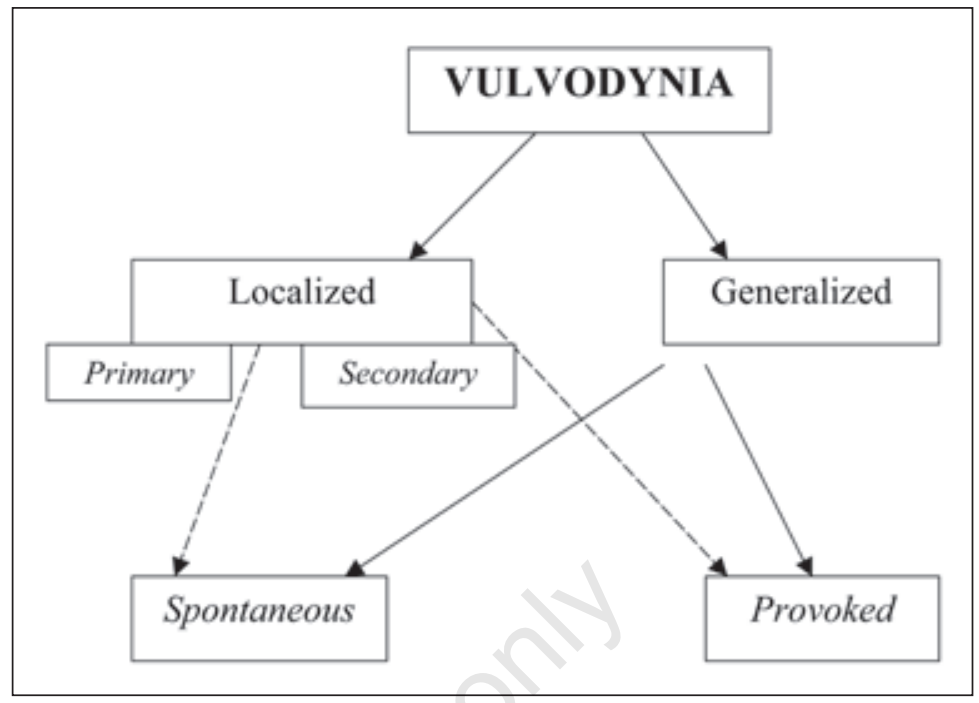

Figure 1

edema with redness of the mucosa) that must be performed delicately and consider particularly urethra (anteriorly, posteriorly and laterally), major and minor labia, perineum, clitoris and vestibule (13).

The painful areas of the vestibular mucosa can be detected by applying a gentle pressure using a normal cotton swab (Q-tip test) clockwise at 2, 4, 6, 8 and 10 o'clock. Each point is assigned a score ranging from 0 to $5(0=$ no pain and $5=$ the greatest elicited pain). When pain is reported only in the posterior region of the vestibule, it is most frequently due to hypertonus of the levator ani (13).

The examination continues with the introduction of a speculum to rule out the presence of interior lesions or sores, and finally a one-finger digital exploration to highlight painful, hypertonic or trigger points that may suggest pelvic floor dysfunction.

The clinical symptoms consist of a burning sensation and sharp pain that may occur spontaneously or after slight pressure. The onset of pain after slight pressure in the vulvar area affects the patients' sexual life by leading to the expectation of pain, avoidance and loss of interest, and subsequent disagreements between the couple (14).

Given this disease probably has the same etiopathogenesis as fibromyalgia, therapy is multifactorial and based on pharmaco- 
logical and non-pharmacological strategies and compliance with adequate standards of hygiene and behavior. The pharmacological strategies include topical preparations, such as local anesthetics and/or the submucosal infiltration of local anesthetics associated with steroids (triamcinolone and local anesthetics); drugs that act on the pain mediator, such as tricyclic antidepressants, anticonvulsants, selective serotonin reuptake inhibitors and selective serotonin and noradrenalin reuptake inhibitors (1519). The non-pharmacological strategies are represented by rehabilitation of the pelvic floor muscles and/or biofeedback techniques involving particularly the levator ani, transcutaneous electrical nerve stimulator, vaginal dilators, and cognitivebehavioral therapy (20-22).

\section{CONCLUSIONS}

The results of a collaborative study carried out by the Rheumatology and Obstetrics/ Gynecology Unit of the University of Siena's Medicine, Surgery and Neurosciences Department (4) seem to show that the frequency of vulvodynia is higher among women with chronic musculoskeletal pain than in the general population $36.7 \%$ vs $16 \%$ ), although they report less intense pain and sexual dysfunction than women with vulvodynia alone.

The epidemiological data confirms the need to take a patient history and conduct a physical examination in order to identify the presence of comorbidities that often play a role in the development and self-renewal of the painful symptoms and account for the number of treatment failures in patients with central sensitization syndromes. The intensity of vulvar pain is more difficult to explain: it appears to be less intense in women with chronic musculoskeletal than in those with vulvar pain alone, and probably involves not only local predisposing factors, but also individual psychological and behavioral factors related to different levels of central sensitization, There is no doubt that a better understanding of the complex neuropsychological and neuroplastic mechanisms underlying the development of central sensitization syndromes would improve our understanding of vulvodynia.

\section{REFERENCES}

1. Vercellini P, Somigliana E, Viganò $\mathrm{P}$, Abbiati A, et al. Chronic pelvic pain in women: etiology, pathogenesis and diagnostic approach. Gynecol Endocrinol. 2009; 25: 149-58.

2. Vincent K. Chronic pelvic pain in women. Postgrad Med J. 2009; 85: 24-9.

3. Dalpiaz O, Kerschbaumer A, Mitterberger $\mathrm{M}$, Pinggera $\mathrm{G}$, et al. Chronic pelvic pain in women: still a challenge. BJU Int. 2008; 102: 1061-5.

4. Ghizzani A, Di Sabatino V, Suman AL, Biasi $\mathrm{G}$, et al. Pain symptoms in fibromyalgia patients with and without provoked vulvodynia. Pain Res Treat. 2014; 2014: 457618.

5. Moyal-Barracco M, Lynch PJ. 2003 ISSVD terminology and classification of vulvodynia: a historical perspective. J Reprod Med. 2004; 49: 772-7.

6. Haefner HK, Collins ME, Davis GD, Edwards $\mathrm{L}$, et al. The vulvodynia guideline. J Low Genit Tract Dis. 2005; 9: 40-51.

7. Harlow BL, Stewart EG. A population-based assessment of chronic unexplained vulvar pain: have we underestimated the prevalence of vulvodynia? J Am Med Womens Assoc. 2003; 58: 82-8.

8. Goldstein AT, Burrows L. Vulvodynia. J Sex Med. 2008; 5: 5-14.

9. Pukall CF, Binik YM, Khalifé S, Amsel R, Abbott FV. Vestibular tactile and pain thresholds in women with vulvar vestibulitis syndrome. Pain. 2002; 96: 163-75.

10. Friedrich EG Jr. Vulvar vestibulitis syndrome. J Reprod Med. 1987; 32: 110-4.

11. Kahn BS, Tatro C, Parsons CL, Willems JJ. Prevalence of interstitial cystitis in vulvodynia patients detected by bladder potassium sensitivity. J Sex Med. 2010; 7: 996-1002.

12. Butrick CW, Sanford D, Hou Q, Mahnken JD. Chronic pelvic pain syndromes: clinical, urodynamic, and urothelial observations. Int Urogynecol J Pelvic Floor Dysfunct. 2009; 20: 1047-53.

13. Goldstein AT, Burrows L. Vulvodynia. J Sex Med. 2008; 5: 5-14.

14. Arnold LD, Bachmann GA, Rosen R, Kelly S, Rhoads GG. Vulvodynia: characteristics and associations with comorbidities and quality of life. Obstet Gynecol. 2006; 107: 617-24.

15. Zolnoun DA, Hartmann KE, Steege JF. Overnight $5 \%$ lidocaine ointment for treatment of vulvar vestibulitis. Obstet Gynecol. 2003; 102: $84-7$. 
16. Steinberg AC, Oyama IA, Rejba AE, KelloggSpadt S, Whitmore KE. Capsaicin for the treatment of vulvar vestibulitis. Am J Obstet Gynecol. 2005; 192: 1549-53.

17. Walsh KE, Berman JR, Berman LA, Vierregger K. Safety and efficacy of topical nitroglycerin for treatment of vulvar pain in women with vulvodynia: a pilot study. J Gend Specif Med. 2002; 5: 21-7.

18. Boardman LA, Cooper AS, Blais LR, Raker CA. Topical gabapentin in the treatment of localized and generalized vulvodynia. Obstet Gynecol. 2008; 112: 579-85.

19. Johannesson U, Sahlin L, Masironi B, Hilliges $\mathrm{M}$, et al. Steroid receptor expression and morphology in provoked vestibulodynia. Am J Obstet Gynecol. 2008; 198: 311.e1-6.

20. Hartmann D, Strauhal MJ, Nelson CA. Treatment of women in the United States with localized, provoked vulvodynia: practice survey of women's health physical therapists. J Reprod Med. 2007; 52: 48-52.

21. Glazer HI, Rodke G, Swencionis C, Hertz R, Young AW. Treatment of vulvar vestibulitis syndrome with electromyographic biofeedback of pelvic floor musculature. J Reprod Med. 1995; 40: 283-90.

22. Hibner M, Desai N, Robertson LJ, Nour M. Pudendal neuralgia. Minim Invasive Gynecol. 2010; 17: 148-53. 